Aus der Sinntalklinik Bad Brückenau

Rehabilitations-Klinik für Innere Medizin,

Schwerpunktklinik für Nephrologie der LVA Unterfranken

AHB-Klinik für Nieren- und Urogenitalerkrankungen

(Chefarzt: Prof. Dr. med. E. Fritschka)

\title{
Langzeiteffekt einer multidisziplinären Schulung bei Patienten mit chronischer Niereninsuffizienz auf Nierenfunktion und renale Risikofaktoren
}

\author{
Inaugural-Dissertation \\ zur \\ Erlangung der medizinischen Doktorwürde \\ des Fachbereichs Humanmedizin \\ der Freien Universität Berlin
}

Vorgelegt von: Jarmila Mahlmeister

Aus:

Ukkel 
Referent: $\quad$ Prof. Dr. med. E. Fritschka, Freie Universität Berlin

Korreferent: Prof. Dr. med. G. Oppermann, Freie Universität Berlin

Promotionsdatum: 12.12.2003. 